\title{
Femtosecond carrier dynamics in bulk graphite and graphene paper
}

\author{
F. Carbone ${ }^{\mathrm{a}, *}$, G. Aubock ${ }^{\mathrm{a}}$, A. Cannizzo ${ }^{\mathrm{a}}$, F. Van Mourik ${ }^{\mathrm{a}}$, R.R. Nair ${ }^{\mathrm{b}}$, A.K. Geim ${ }^{\mathrm{b}}$, \\ K.S. Novoselov ${ }^{\text {, }}$ M. Chergui ${ }^{\text {a }}$ \\ ${ }^{a}$ Laboratory of Ultrafast Spectroscopy, ISIC, Ecole Polytechnique Fédérale de Lausanne, CH-1015 Lausanne, Switzerland \\ ${ }^{\mathrm{b}}$ School of Physics and Astronomy, University of Manchester, Manchester M13 9PL, UK
}

\section{A R T I C L E I N F O}

Article history:

Received 10 December 2010

In final form 19 January 2011

Available online 22 January 2011

\begin{abstract}
A B S T R A C T
Femtosecond white-light continuum probe transient absorption and reflectivity measurements of bulk graphite and graphene paper are reported. In graphite, the relaxation of photoinduced electron-hole pairs happens through in-plane electron-electron and electron-phonon scattering in $\simeq 200 \mathrm{fs}$, while the ps dynamics is due to the modulation of the electronic structure by out-of-plane structural motions. The ps dynamics of the optical signal is strongly reduced in graphene paper, where the out-of-plane bond is disrupted, while the short component of the dynamics is identical in both materials. These results show that in 2D-graphene, the carrier relaxation occurs in $\simeq 200 \mathrm{fs}$.
\end{abstract}

(c) 2011 Elsevier B.V. All rights reserved.
In graphene, the first isolated 2D solid [1], two-dimensionality is responsible for its extraordinary high carrier mobility. Its transport properties promise to revolutionize future microelectronic and optical devices. For instance, it has been shown that the ultrafast carrier relaxation in graphene can be exploited in very efficient saturable absorbers for mode-locking fiber lasers [2]. The improved efficiency of such a device was ascribed to the faster optical response of single layer graphene compared to thicker graphite films. The microscopic phenomena that underline the different response of carriers in low dimensionality conditions are still unknown, and even the scattering mechanisms that undergo photo-excited carriers in bulk graphite are still under debate $[3,4]$. This information is of great importance, not only for the fundamental description of semi-metals [3,4], but also for its technological implications [2]. In fact, the interaction between truly isolated graphene and substrates, or other perturbations to its ideal bi-dimensionality gives rise to carrier-scattering phenomena that can spoil its electronic properties [5]. Thus, a microscopic understanding of such scattering effects is pivotal for the development of graphene-based electronic and optical devices.

A prerequisite for understanding the ultrafast properties of graphene, is a detailed description of those of bulk graphite. Surprisingly, despite years of research, a consensus on the microscopic interpretation of the observed ultrafast dynamics is still lacking. Transient absorption pump-probe studies revealed that the electronic excitation decays in a bi-exponential fashion with an ultrafast ( $\simeq 200 \mathrm{fs}$ ) component and a slower $(1-2 \mathrm{ps}$ ) one $[3,6,7]$. The latter has been interpreted as a slow electron-hole recombination akin to that of a semiconductor [3]. In graphite, infrared fs excita-

\footnotetext{
* Corresponding author.

E-mail address: fabrizio.carbone@epfl.ch (F. Carbone).
}

tion induces strong coherent phonons $[8,9]$ and a sequence of compressions/expansions of the interlayer distance observed by Ultrafast Electron Crystallography (UEC) [10,11]. This translates at early times into a drop of the bulk plasmon contribution concomitant with an increase of the surface plasmon in femtosecond resolved electron energy loss spectroscopy (FEELS) [12,13]. A recent theoretical contribution confirmed that the ps component of the transient optical signal is due to such strong out-of-plane structural distortions launched in graphite by the initial induced charge redistribution [4]. In this scenario, the decay of the electron-hole pairs happens very quickly through in-plane electronelectron and electron-phonon scattering (few hundreds fs), while the following ps dynamics of the optical signal is due to anharmonic couplings involving mainly the $c$-axis lattice motions modulating an already equilibrated electronic structure [4].

A direct confirmation of this scenario would be to carry out pump-probe studies on isolated free-standing graphene, since the $c$-axis ps structural motions should be suppressed; however, this is not yet experimentally feasible. Recent interest has been triggered by the properties of turbostratic graphite [14] and its latest evolution, graphene paper [15]. These systems have an average interlayer distance increased by as much as 30\% and handle like conventional graphite while displaying physical properties closer to those of graphene [15]; for this reason, they are very promising for applications and provide an ideal environment for studying the effect of interlayer scattering on the ultrafast properties of the conduction band carriers in graphite and graphene.

Here, we present new pump-probe experiments with white light continuum on graphite and graphene paper. Our experiments extend previous studies both in spectral region and in fluence (up to those of permanent damage, $170 \mathrm{~mJ} / \mathrm{cm}^{2}$ [16]). The ultrafast optical response of graphene paper is in addition reported for the 
first time. We show that the relaxation of the transient optical signal in graphite and graphene paper decays in a bi-exponential fashion, with a fast ( $\simeq 200 \mathrm{fs}$ ) component associated to in-plane electron-electron and electron-phonon scattering, and a slower one $(\simeq 2 \mathrm{ps})$ associated to the modulation of the electronic structure by out-of-plane structural motions. The latter is significantly weaker in graphene paper, due to the much larger average interlayer distance in this system, which makes it closer to isolated graphene. From these data, we deduce that the characteristic time for the relaxation of the electronic excitation in single layer graphene is $\simeq 200 \mathrm{fs}$.

A thin sample of graphite was prepared by repeatedly cleaving a thick crystal down to $100 \mathrm{~nm}[12,13]$. The film was deposited on a TEM grid with $50 \mu \mathrm{m}$ holes for optical transmission experiments. The reflectivity experiments were instead performed on a bulk natural single crystal of graphite and on a thick (several $\mu \mathrm{m}$ ) graphene paper sample [17]. Graphene paper was prepared as follows: HOPG crystals with a level of paramagnetic impurities as low as $1 \mathrm{ppm}$ were exfoliated by extensive sonication. The obtained suspensions were centrifuged to obtain stable solutions. These solutions were filtered through alumina meshes, resulting in the deposition of graphene laminates several $\mu \mathrm{m}$ thick. Very high purity solvants were used and the purity of the final laminates was better than $0.1 \mathrm{ppm}$.

The specimen was pumped at $\lambda=800 \mathrm{~nm}(1.55 \mathrm{eV})$, and a repetition rate of $500 \mathrm{~Hz}$ with a fluence varying between 5 and $150 \mathrm{~mJ} /$ $\mathrm{cm}^{2}$. The very low repetition rate of our experiment $(500 \mathrm{~Hz}), \mathrm{com}-$ pared to previous studies using $5 \mathrm{kHz}$ to $\mathrm{MHz}$ repetition rates [3,6,9,7], corresponds to $2 \mathrm{~ms}$ between pulses, which allows graphite to dissipate the heat deposited by the intense laser excitation and study its ultrafast optical properties up to the level of permanent damage [16]. The white light continuum probe (1.7-3 eV) was generated in $\mathrm{a} \mathrm{CaF}_{2}$ plate, and directed perpendicular to the graphene layers ( $c$-axis), with the polarization in the graphene planes ( $a b$-plane); the reflected or transmitted signal was recorded on a spectrometer. The overall resolution of the set-up was $40 \mathrm{fs}$ (FWHM) [18].

Figure 1a shows the fluence dependent transient absorption of graphite at a probe energy of $1.7 \mathrm{eV}$. At early times, we find a very fast transmission increase ( $<40 \mathrm{fs}$ ), followed by a bi-exponential decay with an ultrafast component $(\simeq 200 \mathrm{fs})$ and a slower one ( $\simeq 2 \mathrm{ps}$ ), consistent with previous reports at different energies and fluences $[3,6,7]$. At high fluence we also observe a modulation of $\simeq 700 \mathrm{fs}$, due to an out-of-plane coherent phonon [6,9].

Figure $1 \mathrm{~b}$ shows the fluence dependence at 50 fs time-delay for both low (filled black circles, reproduced from [3]), and high excitation (black triangles) which is linear throughout. We also show the fluence dependence of the maximum reflectivity change at $50 \mathrm{fs}$ in both graphite and graphene paper, which has the same slope as the transmission changes for both samples. The fluence dependence of the slower (ps) component, taken at $200 \mathrm{fs}$ time delay, blue open diamonds in Figure 1b, has a weaker slope than the fluence dependence of the maximum transmission/reflection change. The fluence dependence of the contrast between the two components can be estimated by taking $T(\tau=50 \mathrm{fs})-T(\tau=200 \mathrm{fs})$, which has the steepest slope, green squares in Figure $2 \mathrm{~b}$. Because the two components of the TA signal have different fluence dependencies, their relative behavior is more evident at high excitation densities. This observation supports the idea that the two components of the transient optical signal have a different origin.

In Figure 2, the time/energy dependence of the transient reflectivity signal in graphite and graphene paper are shown for an excitation fluence of $150 \mathrm{~mJ} / \mathrm{cm}^{2}$. The data are normalized, and contour lines at given reflectivity changes are plotted for both samples. The overall dynamics of the optical reflectivity is faster in graphene paper than in graphite. Both samples exhibit a strong ultrafast component and a slower one; while at shorter times the dynamics for the two samples are very similar, the slow component in graphene paper appears to be much weaker than in graphite.

The normalized transient reflectivity of graphite and graphene paper at $1.7 \mathrm{eV}$ are displayed in Figure 3 for a pump fluence of $150 \mathrm{~mJ} / \mathrm{cm}^{2}$. The signal from both samples recovers precisely to zero in a few ps, but the relaxation is faster in graphene paper than in graphite. In Ref. [4], the long term dynamics of the optical signal was ascribed to the out-of-plane structural instabilities. As in graphene paper the out-of-plane bond is disrupted, such a component is strongly reduced, and consequently, a faster recovery of the optical transient is expected. In the inset of Figure 3, we zoom into the first 500 fs of the dynamics, and notice that in graphene paper a $\simeq 50$ fs modulation is observed which we attribute to the excitation of one of the strongly coupled optical modes (SCOPS, whose energies range between 90 and $200 \mathrm{meV}$ ). Strong electron-phonon coupling effects in low dimensions have been recently observed also by static optical spectroscopy [19]. We notice that both TA and TR give similar dynamics, in which the decay of the electronic excitation is composed of an ultrafast component ( $\simeq 200 \mathrm{fs})$ and a slower one (in the ps regime), consistent with previous reports $[6,7,3]$.

At very high fluence, the contrast of the out-of-plane coherent phonon, attributed to the shearing motion of the graphene sheets [6], is enhanced in TA geometry (see Figure 1a). This is because the $200 \mathrm{fs}$ component of the TA increases with a higher rate than the slow ps one as a function of fluence, and the contrast between them is enhanced at high pump power, Figure $1 \mathrm{~b}$. In reflection
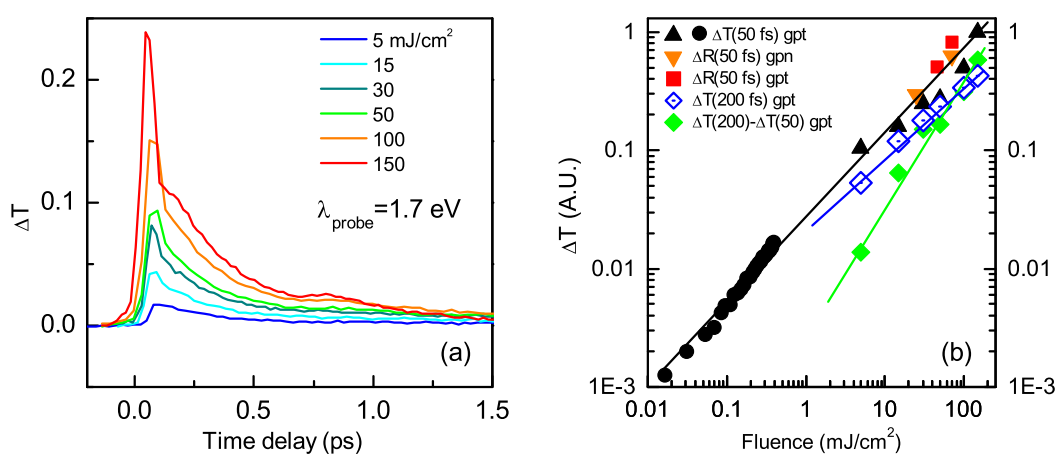

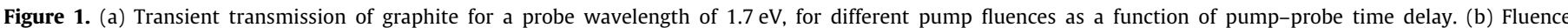

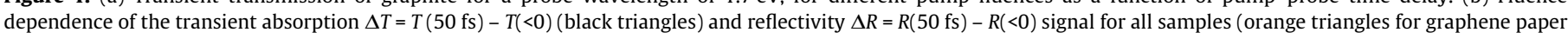

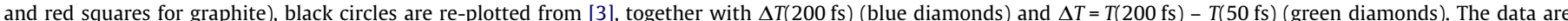

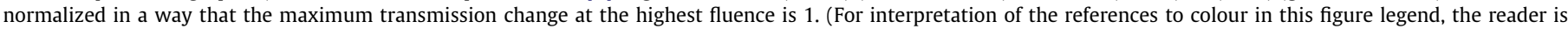
referred to the web version of this article.) 

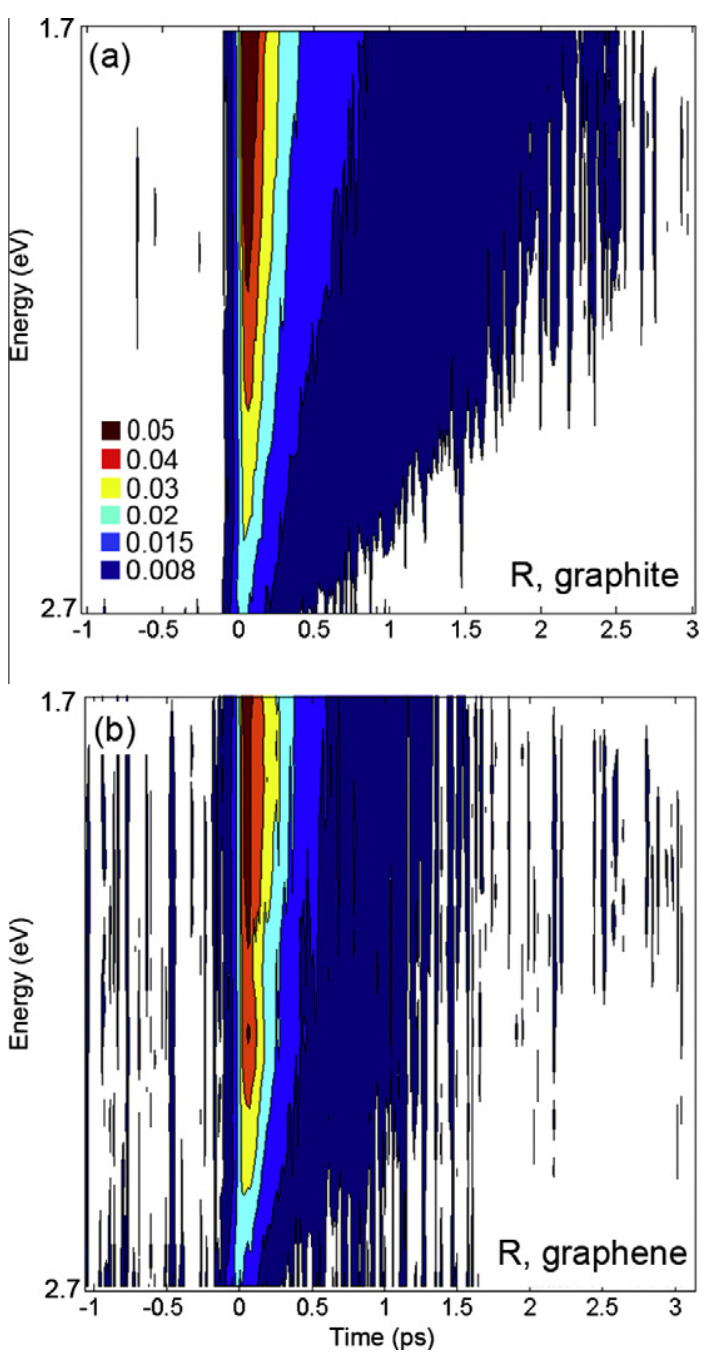

Figure 2. (a) Time-energy dependence of the transient reflectivity in graphite for a pump fluence of $150 \mathrm{~mJ} / \mathrm{cm}^{2}$. (b) Time-energy dependence of the transient reflectivity in graphene paper for a pump fluence of $150 \mathrm{~mJ} / \mathrm{cm}^{2}$.

geometry instead, the contrast to an in-plane coherent phonon is enhanced, (see the inset of Figure 3).

In order to rationalize these results, we fit the transient reflectivity data by a bi-exponential function for all probe wavelengths: $A \cdot e^{-t / \tau_{1}}+B \cdot e^{-t / \tau_{2}}$. The energy dependence of the fitting parameters is displayed in Figure 4. Neglecting the recurrence at $50 \mathrm{fs}$ in graphene paper, the transient data for both samples can be well fitted with two time constants of $\simeq 200 \mathrm{fs}$ and $\simeq 2 \mathrm{ps}$, which are rather probe wavelength-independent (Figure $4 \mathrm{c}$ ). The pre-exponential factor of the fast component has an almost identical behavior in both samples, confirming the idea that it is representative of the carrier dynamics in the single graphene sheets. Instead, the energy dependence of B shows a distinct behavior for graphite and graphene paper, and is much weaker in the latter especially at low energy. Its energy dependence is consistent with that calculated for the optical transmission changes of a $100 \mathrm{~nm}$ graphite film induced by out-of-plane structural dynamics (Figure 3b of Ref. [4]). The decrease in the photoinduced changes at higher wavelengths is due to the suppression of the transmissivity of the material due to the proximity of a strong absorption in the material around $4 \mathrm{eV}$ $[4,20]$. The reduced but still present slow component in the ultrafast optical response of graphene paper is due to the fact that in this system only a fraction (around 50\%) of truly single layer graphene is found.

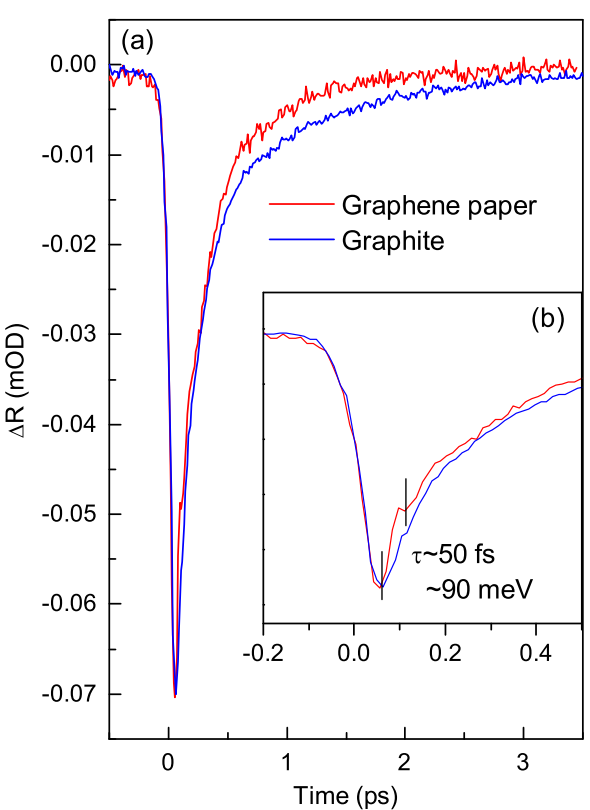

Figure 3. (a) Transient reflectivity for a probe energy of $1.7 \mathrm{eV}$ for graphite and graphene at a fluence of $150 \mathrm{~mJ} / \mathrm{cm}^{2}$

This analysis supports the microscopic scenario presented in [4] and the consequent predictions: (i) the long term dynamics of the transient optical absorption in graphite has a structural origin, and (ii) the relaxation of carriers in single layer graphene is ultrafast, i.e. 200 fs.

In graphite, infrared fs pulses induce an electron-hole plasma which can decay solely exciting certain in-plane phonon modes, called strongly coupled optical phonons (SCOPS) $[8,21,22]$. During the coherence time of these oscillations [9], an ultrafast compression of the $c$-axis followed by a strong lattice expansion was observed by ultrafast electron diffraction[10,11,23]. The coherent vibration of in-plane modes is disrupted by anharmonic couplings

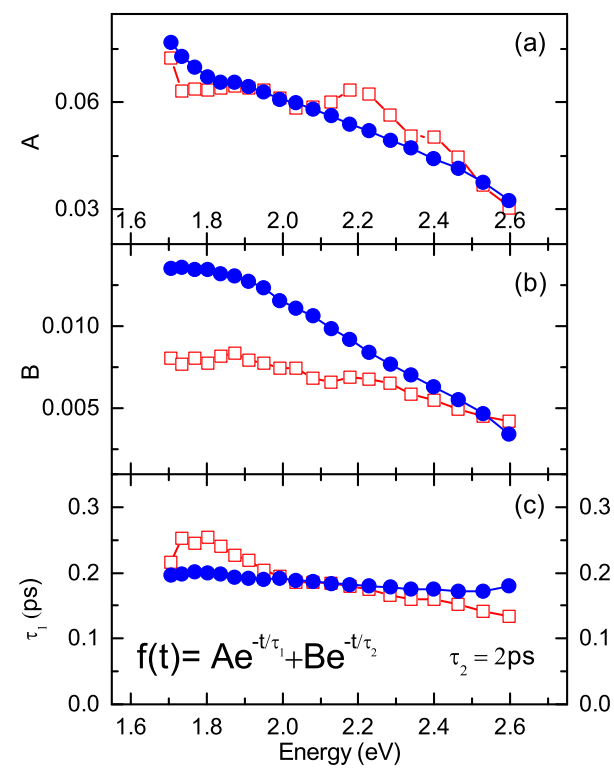

Figure 4. Energy dependence of the fit parameters of the transient reflectivity fitted to a sum of two exponential decays $A \cdot e^{-t / \tau_{1}}+B \cdot e^{-t / \tau_{2}}$. (a) Energy dependence of the weight of the faster component for both samples, $A$. (b) Energy dependence of the weight of the slower component for both samples, $B$. (c) Time constant of the faster component for both samples, $\tau_{1}$. 
to all phonons. When coupling to $c$-axis phonons is suppressed by low dimensionality, as in graphene paper, a more efficient coupling to the SCOPS is expected. This is consistent with our observation of a $50 \mathrm{fs}$ recurrence in the transient reflectivity of graphene paper which is not observed in graphite, Figure $3 \mathrm{~b}$.

By combining the compelling theoretical and experimental evidence that the ultrafast ( $\simeq 200 \mathrm{fs}$ ) carrier dynamics of graphite is governed by in-plane electron-electron and electron-phonon scattering $[3,24,8,10]$, with the results of FEELS [12,13], UEC [10] experiments, $a b$ initio calculations and optical experiments [4], we conclude that the longer ps dynamics is instead due to outof-plane structural effects, and we separate these two regimes: the ultrafast one $(\simeq 200 \mathrm{fs})$ characteristic of the charge carriers response of single graphene layers, and the slower one associated to the three-dimensionality of the material. On the contrary, if the ps dynamics of graphite were governed by electronic effects [3], suppressing scattering channels in low dimensions should induce a longer life-time for the electron-hole pairs, contrary to our observations, and to other recent reports [25,2]. Moreover, the distinct behavior of the two components of the optical transient signal at high fluence supports the idea that they originate from different scattering mechanisms.

Similar arguments were used to extract the static optical response of graphene from that of bulk graphite [20]. By separating the contribution to the optical response of the interaction between layers, the optical conductivity per graphene sheet was found to have a universal value, solely dependent on fundamental constants [20]. This finding was later confirmed by direct measurements of free-standing graphene samples [26]. Such a clear separation between the in-plane and out-of-plane scattering mechanisms in graphite is due to its highly anisotropic electronic structure. Therefore, it is not surprising that it is possible to predict the dynamical behavior of single layer graphene from that of bulk graphite. In fact, our data on graphene paper, together with those in [25,2], confirm this point and give a quantitative estimate of the carrier dynamics in isolated graphene.
In conclusion, the new ultrafast optical spectroscopy on graphite and graphene paper, combined with the knowledge of the structural dynamics obtained by UEC [10], fs EELS[12,13], and $a b$ initio calculations [4] allowed us to unravel the dynamical response of graphite in detail. Assessing the different decay channels for excited carriers, we find that the carrier dynamics in graphite are governed by the physics of the single graphene sheets at early times and by the interlayer structural dynamics at later times.

The authors thank to Prof. A. Zewail and Dr. A. Kuzmenko for stimulating discussions. FC acknowledges support from the Swiss National Science Foundation.

\section{References}

[1] K.S. Novoselov et al., Science 306 (2004) 666

[2] Q. Bao et al., Phys. Opt. (2010). 1007.2243.

[3] M. Breusing, C. Ropers, T. Elsaesser, Phys. Rev. Lett. 102 (2009) 086809.

[4] F. Carbone, Chem. Phys. Lett. 496 (2010) 291.

[5] G.M. Rutter et al., Science 317 (2007) 219.

[6] T. Mishina, K. Nitta, Y. Masumoto, Phys. Rev. B 62 (2000) 2908.

[7] A.M. Malvezzi, N. Bloembergen, C.Y. Huang, Phys. Rev. Lett. 57 (1986) 146.

[8] R. Saito et al., Phys. Rev. Lett. 88 (2001) 027401.

[9] K. Ishioka et al., Phys. Rev. B 77 (2008) 121402(R).

[10] F. Carbone, P. Baum, P. Rudolf, A.H. Zewail, Phys. Rev. Lett. 100 (2008) 035501

[11] R.K. Raman et al., Phys. Rev. Lett. 101 (2008) 077401.

[12] F. Carbone et al., Chem. Phys. Lett. 468 (2008) 107.

[13] F. Carbone, O.H. Kwon, A.H. Zewail, Science 325 (2009) 181.

[14] Z. Kurban et al., Eur. Polym. J. 46 (2010) 1194.

[15] D.A. Dikin et al., Nature 448 (2007) 457.

[16] H.O. Jeschke, M.E. Garcia, K.H. Bennemann, Phys. Rev. Lett. 87 (2001) 015003.

[17] P. Blake et al., Nano Lett. 8 (2008) 1704.

[18] J. Helbing et al., Biophys. J. 87 (2004) 1881.

[19] E. Cappelluti, L. Benfatto, A.B. Kuzmenko, Phys. Rev. B. 82 (2010) 041402R.

[20] A.B. Kuzmenko, E. Van Heumen, F. Carbone, D. Van der Marel, Phys. Rev. Lett. 100 (2008) 117401.

[21] T. Kampfrath, L. Perfetti, F. Schapper, C. Frischkorn, M. Wolf, Phys. Rev. Lett. 95 (2005) 187403.

[22] G. Moos, C. Gahl, R. Fasel, M. Wolf, T. Hertel, Phys. Rev. Lett. 87 (2001) 267402.

[23] J. Kanasaki, E. Inami, K. Tanimura, H. Ohnishi, K. Nasu, Phys. Rev. Lett. 102 (2009) 087402.

[24] A. Acocella, F. Carbone, F. Zerbetto, J. Am. Chem. Soc. 132 (2010) 12166.

[25] R.W. Newson, J. Dean, B. Schmidt, H.M. Van Driel, Opt. Express 17 (2009) 2326.

[26] K.F. Mak et al., Phys. Rev. Lett. 101 (2008) 196405. 